\title{
LOS ESTILOS DE APRENDIZAJE: UNA PROPUESTA PEDAGÓGICA PARA OPTIMIZAR LA ENSEÑANZA DE LAS LENGUAS EXTRANJERAS EN LA LICENCIATURA EN LENGUAS MODERNAS DE LA UNIVERSIDAD DEL QUINDÍO*
}

\author{
LEARNING STYLES: A PEDAGOGICAL PROPOSAL TO ENHANCE FOREIGN LANGUAGE \\ TEACHING IN LICENCIATURA EN LENGUAS MODERNAS AT UNIVERSIDAD DEL QUINDÍO
}

Neira Loaiza Villalba ${ }^{1}$ A Alexis Guevara Henao ${ }^{1}$

${ }^{1}$ Programa de Lenguas Modernas, Universidad del Quindío. Correo electrónico: neiraloaiza@hotmail. com, alexisgh1@hotmail.com.

Recibido: POR CONFIRMAR

Aceptado: POR CONFIRMAR

*Correspondencia del autor: Programa de Lenguas Modernas. Universidad del Quindío. Av. Bolivar calle 12 Norte Armenia Quindío Colombia. e-mail: neiraloaiza@hotmail.com

\begin{abstract}
RESUMEN
Este artículo presenta los resultados de la investigación titulada,"Los estilos de aprendizaje: una propuesta pedagógica para optimizar la enseñanza de las lenguas extranjeras en la Licenciatura en Lenguas Modernas de la Universidad del Quindío". Dicha investigación, no experimental, transversal y exploratorio-descriptiva, se inscribe en el campo de la teoría de los estilos de aprendizaje y su aplicación como herramienta pedagógica. Su finalidad fue identificar, mediante la aplicación de los inventarios de estilos de aprendizaje del modelo de Felder y Silverman y del de Herrmann, los estilos de aprendizaje de estudiantes y, mediante el inventario de Herrmann para docentes, el estilo personal de enseñanza de profesores de los cursos básicos de inglés y francés del mencionado programa. El diagnóstico confirmó la heterogeneidad en los estilos de aprendizaje de los estudiantes y la disimilitud entre los estilos de éstos y el estilo personal de enseñanza de los docentes. Esta investigación arroja como producto algunos lineamientos que sirven para la posterior estructuración de una propuesta pedagógica de secuencias didácticas que optimice la enseñanza y el aprendizaje en este campo del saber y que puede guiar el diseño curricular a partir de las implicaciones pedagógicas que los estilos de aprendizaje de los estudiantes y el estilo personal de enseñanza de los docentes tienen para el acto educativo.
\end{abstract}

PALABRAS CLAVES: estilos de aprendizaje, estilos de enseñanza, aprendizaje, propuesta pedagógica, diseño curricular.

\footnotetext{
"Este artículo se inscribe en la línea de investigación Enseñanza y aprendizaje de lenguas extranjeras del Grupo de Investigación Estilos de Aprendizaje e Idiomas Extranjeros y Bilingüismo (ESAPIDEX-B) de la Universidad del Quindío en Colombia (Categoría C en Colciencias). Es, igualmente, el resultado del proyecto de investigación No 502, aprobado y auspiciado por la Vicerrectoría de Investigaciones de esta Universidad y realizado entre 2010-2 y 2011-2. Participaron en él los investigadores Neira Loaiza, Alexis Guevara y Norma Díaz. El proyecto contó con la colaboración de la estudiante Sandra Chisco, auxiliar de investigación
} 


\begin{abstract}
This article presents the results of the research entitled, "Learning styles: a pedagogical proposal to enhance foreign language teaching in Licenciatura en Lenguas Modernas at Universidad del Quindío."This research, non-experimental, transversal and exploratory-descriptive, falls within the realm of the theory of learning styles and its application as a teaching tool for the optimization of the processes of teaching and learning of foreign languages. Its purpose was to identify, by applying Felder and Silverman's and Herrmann's questionnaires, the learning styles of students; and, by applying Herrmann's questionnaire for teachers, the personal teaching styles of teachers in the basic French and English courses at the Modern Languages Program in Universidad del Quindío. The diagnosis confirmed the heterogeneity of students' learning styles and the dissimilarity between these styles and the teachers' personal teaching styles. This research provides, as a product, some pedagogical guidelines for the future structuring of a pedagogical proposal of didactic sequences that might enhance teaching and learning in this field of study and might eventually be of use in curriculum design, based on the implications of the learning and teaching styles detected.
\end{abstract}

KEYWORDS: Learning styles, teaching styles, learning, pedagogical proposal, and curriculum design.

\section{INTRODUCCIÓN}

Esta investigación educativa ${ }^{\dagger}$ se fundamenta en la teoría de los estilos de aprendizaje y los beneficios que su aplicación aporta tanto a docentes como a estudiantes en los procesos de enseñanza y aprendizaje de las lenguas extranjeras. Dicha teoría se cimenta en el hecho de que los seres humanos tienen sensibles diferencias a nivel cognitivo, afectivo y fisiológico en la manera de acceder al conocimiento. Por ello, el diseño de una propuesta pedagógica que se sustente en este planteamiento científico contribuirá seguramente en el diseño curricular y en el desarrollo y fortalecimiento de las competencias lingüísticas y comunicativas de los estudiantes de lenguas. A nivel teórico, la investigación se fundamenta en los conceptos de estilo de aprendizaje, sus beneficios para docentes y estudiantes, la aplicación de la teoría de estilos de aprendizaje en el campo de las lenguas extranjeras, y los estilos de enseñanza, los cuales se presentan de manera sucinta a continuación.

\section{Aproximación teórica al concepto de Estilo de Aprendizaje}

La teoría de estilos de aprendizaje, al decir de Keefe (1988, en Alonso, Gallego y Honey, 1994), son los rasgos cognitivos, afectivos y fisiológicos que sirven como

\footnotetext{
$\dagger$ Esta investigación se considera educativa de acuerdo con lo que plantean Latorre, del Rincón y Arnal (1996) acerca de este tipo de investigación, cuya intención es "generar conocimiento útil para la acción educativa, ya se trate de una acción política o de un cambio en la práctica educativa (Keeves, 1988); se erige pues como guía de la acción educativa". Estos autores mencionan una serie de características de la investigación educativa, entre ellas, su carácter complejo, pluriparadigmático, plurimetodológico y multidisciplinar.
}

indicadores relativamente estables, de cómo los discentes perciben, interaccionan y responden a sus ambientes de aprendizaje. Los rasgos cognitivos tienen que ver con la forma como los estudiantes estructuran los contenidos, forman y utilizan conceptos, interpretan la información, resuelven los problemas, seleccionan medios de representación (visual, auditivo, kinestésico), etc. Los rasgos afectivos se relacionan con las motivaciones y expectativas, la experiencia previa, las preferencias por los contenidos, asignaturas o temas que influyen en el aprendizaje, y los rasgos fisiológicos están relacionados con el biotipo y el biorritmo del estudiante, los cuales también pueden incidir en la manera como se aprenda. El biotipo se relaciona con las características hereditarias comunes a un grupo de seres vivos, y el biorritmo tiene que ver con el ciclo periódico en que se desarrollan los procesos vitales de una persona o un animal, el cual incidiría en determinados estados de ánimo que se manifiestan cada cierto tiempo.

De igual manera, la persona recibe influencias de los diferentes contextos socioculturales en los cuales está inmersa: desde la familia con sus valores y costumbres, pasando por la escuela y sus concepciones pedagógicas, la religión, el trabajo, la idiosincrasia, las ideologías y cosmogonía, etc., contextos que delinean y definen en gran medida los comportamientos de los individuos.

De acuerdo con Lozano (2009), los estilos de aprendizaje están conformados por elementos tales como disposición, preferencias, tendencia, patrones conductuales, habilidad, estrategia de aprendizaje. Elementos que este autor encuentra como soporte de diferentes modelos teóricos de estilos de aprendizaje que él mismo cita. 
Así, el modelo de Pask (1988) se basa en las disposiciones; el de Stenberg (1977), Hirsh y Kummerow (1990), Dunn y Dunn (1978) y otros autores, en las preferencias y gustos; el de Kagan (1967), en las tendencias e inclinaciones; el de Ryding y Rayner (1998) Guild y Garger (1998), en los patrones conductuales y en las estrategias de aprendizaje; y el de Gardner, en habilidades y fortalezas.

Lozano (2009) define la disposición como el estado físico o intelectual de una persona para realizar o no una acción. Las preferencias como los gustos y posibilidades de elegir entre varias alternativas; las tendencias como la inclinación a ejecutar una acción de cierta manera, sea consciente o inconscientemente; los patrones conductuales como las manifestaciones típicas de un individuo en circunstancias específicas; la habilidad como una capacidad física o intelectual que sobresale en una persona y que se constituye en una fortaleza; y las estrategias de aprendizaje como toda herramienta cognitiva a la que el individuo recurre para solucionar y llevar a feliz término una tarea y cuyo resultado es el conocimiento.

Esta serie de elementos interactúan en la persona, cumplen una función, demarcando su estilo de aprendizaje frente a una tarea específica. El solo gusto, preferencia, tendencia que se muestre no es suficiente para caracterizar el estilo de aprendizaje en un momento determinado, es importante el patrón conductual, que va ligado a rasgos de su personalidad, y que incide en algunas características individuales como su modo de vestir, hablar, pensar, enseñar o aprender. La preferencia no determina de manera intrínseca el estilo pero sí permite identificarlo dado su frecuencia de ocurrencia. Asimismo, el presentar una característica propia de un determinado estilo no asegura de inmediato que se tenga la habilidad, si bien ésta se puede adquirir y desarrollar mediante la práctica frecuente. El hecho de que a una persona le guste la danza y tenga un estilo creativo no implica que automáticamente se convierta en un(a) gran bailarín(a); se necesita además del gusto, la disposición y la creatividad, aprender la técnica y practicarla hasta lograr la habilidad.

\section{Beneficios de la teoría de los estilos de aprendizaje para los docentes}

Labatut Portilho (2004) destaca algunas de los planteamientos de Catalina Alonso, fruto de sus investigaciones acerca de la teoría de los estilos de aprendizaje, que resaltan la importancia y los beneficios que tal teoría reporta a los docentes. Dentro de los aspectos más relevantes puede mencionarse que, debido a la naturaleza de la teoría misma, basada en los rasgos y particularidades de la persona, y a la variedad de sus aplicaciones, ésta puede aprovecharse en todo contexto educativo (educación básica, media, técnica y superior; formal y no formal) permitiendo la individualización de la instrucción. Además, otro aspecto para recalcar es su mutabilidad y adaptabilidad; si bien son estables, no son inmutables, lo que implica la intervención del docente para flexibilizarles, esto es, para enseñar a aprender de maneras diferentes. Por otra parte, consciente de la influencia que tiene el estilo personal de enseñanza sobre el aprendizaje, el docente, ajustando su estilo de enseñanza a la diversidad de estilos de aprendizaje de sus estudiantes, puede potencializar el rendimiento académico, ya que existe una correlación positiva entre estos dos aspectos*.

\section{Beneficios de la teoría de los estilos de aprendizaje para los estudiantes}

Según diversas investigaciones realizadas, la teoría de los estilos de aprendizaje beneficia de diferentes maneras a los estudiantes. Carter y Lyman (1997, citadas en Reyes, 2008), retoman a Soloman:

Con frecuencia, surgen desajustes entre los estilos de aprendizaje comunes y los estilos de enseñanza estándar. Por tanto, a menudo los estudiantes tienen un desempeño ineficiente y se desaniman (...) Si los estudiantes comprenden cómo aprenden con mayor eficiencia, pueden adaptar a sus necesidades su manera de estudiar. Esto no sólo ayudará a aumentar sus calificaciones, sino también a incrementar su autoestima, al darse cuenta de que las dificultades que enfrentan pueden deberse a desajustes entre sus estilos de aprendizaje y los de enseñanza de sus profesores.

El descubrimiento y el entendimiento de sus propios estilos de aprendizaje, según el planteamiento de Soloman, llevará a los estudiantes a mejorar sus procesos de aprendizaje y, consecuentemente, el rendimiento académico; asimismo, optimizando el tiempo involucrado e incrementando también la autoestima al reconocerse que las deficiencias y fracasos al momento de aprender

\footnotetext{
"La relación estilos de aprendizaje y rendimiento académico es una de las temáticas en las que ha profundizado la investigación en torno a los estilos de aprendizaje (Cafferty, 1980; Lynch, 1981; Pizzo, 1981; Krimsky, 1982; White, 1879; Gardner, 1990; Wheeler, 1983; citados en Alonso, Gallego y Honey, 1994).
} 
no se han dado por falta de aptitud sino por desconocimiento del cómo se aprende. Como también lo expresa Reyes (2008):

para los estudiantes resulta difícil controlar o cambiar lo qué sus profesores enseñan y cómo lo enseñan, sin embargo, al identificar sus estilos de aprendizaje, pueden tomar algunas medidas que les permita ayudarse a ellos mismos, por ejemplo, si un estudiante ha identificado, como su estilo de aprendizaje más importante, el estilo secuencial, puede estudiar re-ordenando sus notas de clase de manera que la secuencia que siga para revisar sus apuntes sea la más conveniente para comprender su contenido.

\section{La teoría de los estilos de aprendizaje en la enseñan- za de las lenguas extranjeras}

En el campo de las lenguas en general, las primeras investigaciones sobre estilos de aprendizaje inician hace unas tres décadas en el contexto de la lengua materna. En cuanto al aprendizaje de una segunda lengua o de una lengua extranjera, las investigaciones son aún mucho más recientes y aún quedan muchos campos por explorar. No obstante, existe común acuerdo en cuanto a las ventajas de la aplicación de la teoría para los aprendientes de lengua extranjera. Así lo expresa Hernández (2004), al reconocer que el conocimiento sobre el tema es aún insuficiente, ya que se necesitan más investigaciones e instrumentosadaptados a la población estudiantil. Sin embargo es claro el objetivo de lograr una actitud más positiva y una mayor motivación por parte del estudiante hacia el estudio y simultáneamente mejorar su aprendizaje.

Pese a lo reciente de este tipo de estudios, algunas investigaciones han hecho aportes muy significativos. Dell'Ordine (2001), diseñó un curso piloto de lengua extranjera basado enteramente en el diagnóstico de los estilos de aprendizaje de sus estudiantes (adultos), curso que a su vez recurría a la literatura y las culturas medievales y a una metodología de inmersión. Kindelán (2000), quien estudia los estilos de aprendizaje de una segunda lengua en contextos científicos y tecnológicos, afirma que los estudiantes, al conocer sus estilos de aprendizaje, pueden afrontar el aprendizaje de una lengua extranjera de la manera más efectiva; para ilustrar, el visual recurrirá a material impreso, el auditivo querrá escuchar la lengua, el teórico querrá analizar las estructuras gramaticales. Asimismo lo señalan Alonso, Gallego y otros (1994) cuando afirman que el aprender a aprender constituye la acción subyacente a la autonomía del alumno, lo que implica el autoconocimiento y la reflexión sobre sus mismos procesos; lo que conlleva a mejorar el rendimiento académico de los estudiantes.

Asimismo, Martínez (2002, p. 192), en su investigación, "Tendencias en los estilos de aprendizaje de una lengua extranjera" al analizar las diferentes tendencias de aprendizaje, en cuanto a preferencias sensoriales de los estudiantes concluyó que:

Los datos experimentales confirman estadísticamente la selección de determinadas preferencias de aprendizaje que estarán condicionadas por las exigencias de las necesidades formativas específicas del alumnado. Además, los resultados demuestran que la adopción de uno u otro estilo de aprendizaje están determinados también por el grado de dominio adquirido en las diferentes destrezas o habilidades lingüísticas.

Estos desarrollos en el campo de las lenguas extranjeras reflejan la preocupación de los docentes investigadores para llevar a la praxis pedagógica el reconocimiento de las diferencias individuales de aprendizaje, partiendo del planteamiento de que la identificación, entendimiento y toma de conciencia de los estilos de aprendizaje por parte de los estudiantes y del docente, puede facilitar y dinamizar significativamente el proceso de aprendizaje de una lengua extranjera promoviendo, a la vez, autonomía y responsabilidad en los estudiantes.

\section{Los estilos de enseñanza}

Puesto que el hecho educativo involucra tanto aprendizaje como enseñanza y ésta última tiene como objeto al primero, es cada día más frecuente hablar de estilo personal de enseñanza cuando bien se habla de estilos de aprendizaje. El estilo personal de enseñanza se define, según Rendón y otros $(2010$, p.7) como:

modos, formas, adopciones o maneras particulares y características de pensar el proceso educativo $y$ de asumir el proceso de enseñanza aprendizaje en un contexto específico; es decir, actitudes, comportamientos, acciones, procedimientos, actividades que se ponen en juego en la praxis docente en función de aspectos como: relación, interacción, socialización y orientación de los estudiantes, organización, preparación o planificación de la actividad académica, presentación de la información, dirección, conducción y control del proceso de enseñanza aprendizaje, métodos de enseñanza, dirección de las tareas, ambiente 
de aula, evaluación. Estos son producto de supuestos, principios, creencias, ideas y conceptos subyacentes a las prácticas pedagógicas que pueden ser más o menos conscientes.

Diversas investigaciones al respecto en el contexto iberoamericano (Arce y Estrella, 1998; Pinelo, 2008; De León, 2005; Centeno y otros, 2005) demuestran que, generalmente, no existe semejanza entre los estilos de aprendizaje de los estudiantes y los estilos de enseñanza de los profesores y que los profesores pueden asumir varios estilos de enseñanza; por lo tanto, debe hablarse de tendencias y no de estilos únicos. Además, dentro de la literatura científica, existen pocos instrumentos para la identificación de tales estilos, lo que ha llevado a los investigadores a desarrollar sus propios cuestionarios respaldados por modelos teóricos y explicativos. No obstante, uno de los inventarios más conocidos para la detección de éstos es el de Herrmann, que se fundamenta en el mismo modelo de los cuadrantes cerebrales que describen los estilos de aprendizaje (Herrmann, según Ruiz, 2004).

Las anteriores consideraciones resaltan la importancia de la teoría de los estilos de aprendizaje y la necesidad de que docentes y estudiantes sean conscientes de que el descubrimiento de los rasgos cognitivos, afectivos y fisiológicos que caracterizan el estilo de aprendizaje de los seres humanos es esencial en la comprensión de los procesos que se dan alrededor del hecho pedagógico.

En este sentido, como se ha señalado con anterioridad, esta investigación pretende aportar a la didáctica de las lenguas extranjeras mediante la conceptualización de unos lineamientos, cimentados sobre las implicaciones de los estilos de aprendizaje de estudiantes y de estilos de enseñanza de docentes, que permita la estructuración de una propuesta curricular y pedagógica que optimice la enseñanza y el aprendizaje en ésta área del saber.

\section{METODOLOGÍA}

Esta investigación educativa adoptó un diseño de investigación no experimental, de carácter transversal y exploratorio-descriptivo. Se adopta un diseño no experimental, ya que no hay manipulación de variables, y tal como lo plantean Latorre y otros. (1996), sólo se hacen inferencias sobre relaciones, sin intervención directa de la situación o contexto en el cual se recogen los datos. En este caso, la acción investigativa se enfocó en obtener y analizar información de base para un diagnóstico de los estilos de aprendizaje de los estudiantes y del estilo personal de enseñanza de los docentes de la muestra. A partir del diagnóstico se elaboraron unos lineamientos pedagógicos generales para la estructuración posterior de una propuesta de secuenciación didáctica a implementarse y evaluarse en procesos de investigación continua.

Se adoptó un diseño flexible de carácter esencialmente cualitativo, que permitió y estimuló la realización de ajustes, en particular en la etapa de elaboración de los lineamientos, a fin de aprovechar la información obtenida en la fase diagnóstica. Esta investigación exploratoria se constituye en un acercamiento inicial al tema de los estilos de aprendizaje y de enseñanza en el contexto de un programa de lenguas extranjeras en nuestra región. En este sentido, los resultados y los lineamientos pedagógicos generados para el diseño de una propuesta de secuenciación didáctica con miras a optimizar los cursos de inglés y francés de la Licenciatura en Lenguas Modernas de la Universidad del Quindío, representan una aproximación diferente a la teoría, posible de aplicar en otros contextos similares al nuestro.

Por otra parte, dado que se realizó un estudio diagnóstico, esta investigación también tuvo un alcance descriptivo. Como aseveran Hernández y otros (2010, p. 80) "Los estudios descriptivos buscan especificar las propiedades, las características, y los perfiles de personas, grupos, comunidades, procesos, objetos o cualquier otro fenómeno que se someta a un análisis", y justamente esta investigación buscó trazar los perfiles tanto de los estilos de aprendizaje de un grupo de estudiantes universitarios como del estilo personal de enseñanza de un grupo de sus docentes.

Si bien, la investigación en su segunda etapa (elaboración de los lineamientos pedagógicos generales) fue de carácter cualitativo, en la primera etapa (elaboración del diagnóstico) se emplearon técnicas cuantitativas de análisis de datos de acuerdo con la categoría de asunto o tópico propuesta por Krippendorff (1990), particularmente al caso, la categoría de estilo de aprendizaje y las subcategorías correspondientes a cada uno de los diferentes estilos de aprendizaje y de estilos personales de enseñanza, a partir del análisis de los modelos de Herrmann y de Felder y Silverman. La técnica de análisis de los datos obtenidos fue la de distribución de porcentajes, la cual se representó mediante histogramas. La muestra gozó de un nivel de confianza de $95 \%$ con un margen de error de $5 \%$. 
La muestra estuvo conformada por 116 estudiantes y 16 docentes $(25 \%$ y $90 \%$ de la población, respectivamente) de los cursos básicos de inglés y de francés. Para el diagnóstico de los estilos de aprendizaje, se escogió el inventario de Felder y Silverman y el inventario de Herrmann, y para el diagnóstico de los estilos de enseñanza el inventario que el mismo Herrmann propuso para los docentes.

El modelo de Felder y Silverman hace una clasificación de los estilos de aprendizaje a partir de cuatro escalas bipolares que son: Activo-Reflexivo, Sensorial-Intuitivo, Visual-Verbal y Secuencial-Global. Con base en estas escalas, Felder diseñó un inventario o cuestionario de 44 preguntas que indagan sobre los comportamientos, actitudes y preferencias de los estudiantes en determinadas situaciones o circunstancias.

Herrmann, según Ruiz (2004), basado en los estudios sobre la dominancia cerebral de Sperry, (1973) y en la teoría del cerebro triuno de MacLean (1978) y en sus propias investigaciones, replanteó el problema de la dominancia cerebral, proponiendo la teoría del cerebro total. En este modelo, integra el neocortex (hemisferios derecho e izquierdo) con el sistema límbico, concibiendo el cerebro como una totalidad orgánica dividida en cuatro áreas o cuadrantes que representan cuatro maneras diferentes no solo de aprender sino también de pensar, crear y concebir el mundo: cortical derecho, cortical izquierdo, límbico derecho y límbico izquierdo.

Con base en la teoría de Herrmann, Chalvin (1995) diseñó dos inventarios dirigidos a identificar los estilos de aprendizaje de los estudiantes y el estilo personal de enseñanza de los docentes. Tales inventarios, posteriormente modificados por Pablo Cazau (2004), hacen parte de los instrumentos que se utilizaron para la realización del diagnóstico. El inventario para estudiantes está conformado por doce (12) preguntas que indagan acerca de la vida escolar, la relación con los profesores, los métodos de aprendizaje, el trabajo en grupo, la actitud ante la evaluación, las materias y las lecturas preferidas y los idiomas. Por su parte, el inventario para docentes consta de diez (10) preguntas que indagan sobre la personalidad, la relación con los alumnos y colegas, la preparación de clases, el control de la enseñanza, los soportes pedagógicos, la evaluación del aprendizaje y la actitud hacia la clase.

\section{RESULTADOS Y DISCUSIÓN}

\section{Presentación y análisis de resultados \\ Inventario estilos de aprendizaje de Felder y Silverman aplicado a estudiantes}

De acuerdo con las categorías bipolares de estilos de aprendizajes planteadas por el modelo de Felder y Silverman, la muestra de estudiantes presentó el siguiente comportamiento (Tabla 1).

De acuerdo con los resultados del inventario de Felder y Silverman, se evidencia una muestra mayoritariamente con equilibrio apropiado (EA) entre lo activo y lo reflexivo (59\%), lo sensorial y lo intuitivo (58\%), lo secuencial y lo global (59\%), y un resto de estudiantes con una preferencia moderada (PM) a lo activo (28\%), sensorial $(27.7 \%)$, secuencial $(32 \%) \%$ ), y una proporción muy cercana entre quienes presentan EA entre lo visual y verbal (39\%) y quienes presentan PM por lo visual (38\%).

Esto implica entonces, según la caracterización de Felder y Silverman para cada estilo, que si bien la mayoría de los estudiantes optan por ambientes más estructurados, lógicos, deductivos, secuenciales, regidos por un programa de curso claro y coherente, con medios y objetivos claramente definidos desde un principio, con instrucciones claras, también es cierto que hay otros estudiantes que prefieren ambientes menos estructurados, más informales, que requieren interacción, cooperación y colaboración, que aprecian las innovaciones que se puedan dar en el aula de clase y que aprenden también a través de su propia intuición y reflexión.

\section{Inventario de estilos de aprendizaje de Herrmann apli-} cado a estudiantes

Con respecto al inventario de Herrmann, los resultados reflejan una muestra mayoritaria de estudiantes con preferencia neta (PN) al estilo cortical izquierdo-CI (76\%), seguidos por un grupo (39\%) con PN al estilo límbico derecho-LD, otro grupo de PN en el estilo cortical derecho-CD (36\%) y un cuarto grupo de PN al estilo límbico izquierdo-LI (27\%), siendo posible la dominancia de varios estilos de aprendizaje en un mismo estudiante (Ver tabla 2).

Muy a pesar de que la mayoría de los estudiantes se ubican con preferencia al CI, debe tenerse en cuenta que los resultados son heterogéneos, es decir, existen 
Tabla 1. Resultados del inventario de estilos de aprendizaje de Felder y Silverman aplicado a estudiantes.

\begin{tabular}{|l|c|c|c|c|c|}
\hline $\begin{array}{c}\text { Preferencias } \\
\text { Estilos de } \\
\text { aprendizaje }\end{array}$ & $\begin{array}{c}\text { Equilibrio } \\
\text { apropiado }\end{array}$ & $\begin{array}{c}\text { Preferencia } \\
\text { moderada en } \\
\boldsymbol{a}^{*}\end{array}$ & $\begin{array}{c}\text { Preferencia } \\
\text { moderada } \\
\text { en } \boldsymbol{b}\end{array}$ & $\begin{array}{c}\text { Preferencia } \\
\text { fuerte en } \boldsymbol{a}\end{array}$ & $\begin{array}{c}\text { Preferencia } \\
\text { fuerte en } \boldsymbol{b}\end{array}$ \\
\hline Activo-Reflexivo & $59.6 \%$ & $28.5 \%$ & $6.2 \%$ & $4.2 \%$ & $0.8 \%$ \\
\hline Sensorial-Intuitivo & $58.8 \%$ & $27.7 \%$ & $7.5 \%$ & $5.8 \%$ & $0 \%$ \\
\hline Visual-Verbal & $39.4 \%$ & $38.6 \%$ & $4.2 \%$ & $15.9 \%$ & $1.6 \%$ \\
\hline Secuencial-Global & $59.6 \%$ & $32.7 \%$ & $3.3 \%$ & $4.2 \%$ & $0 \%$ \\
\hline
\end{tabular}

*De acuerdo con las categorías bipolares de Felder y Silverman a es la primera categoría mencionada y $\boldsymbol{b}$, la segunda.

Tabla 2. Resultados del inventario de estilos de aprendizaje de Herrmann aplicado a estudiantes.

\begin{tabular}{|l|c|c|c|c|}
\hline Ereferencia & $\begin{array}{c}\text { Cortical } \\
\text { izquierdo de aprendizaje }\end{array}$ & $\begin{array}{c}\text { Límbico } \\
\text { izquierdo }\end{array}$ & $\begin{array}{c}\text { Límbico } \\
\text { derecho }\end{array}$ & $\begin{array}{c}\text { Cortical } \\
\text { derecho }\end{array}$ \\
\hline Neta (mayor a 66 puntos) & $76.4 \%$ & $27.7 \%$ & $39.4 \%$ & $36.1 \%$ \\
\hline Intermedia (entre 33 y 66 puntos) & $22.6 \%$ & $50.4 \%$ & $46.2 \%$ & $47.8 \%$ \\
\hline $\begin{array}{l}\text { NO preferencia (menor a 33 } \\
\text { puntos) }\end{array}$ & $0.8 \%$ & $21.8 \%$ & $14.2 \%$ & $15.9 \%$ \\
\hline
\end{tabular}

estudiantes, aunque en menores porcentajes, con tendencias marcadas en algunos de los otros cuadrantes. Esta heterogeneidad se ve aún más marcada cuando se toma en cuenta las tendencias intermedias o moderadas, planteando al docente dos implicaciones directas: 1) la diversidad de características, de estilos, de maneras de pensar, de ser, de aprender, de hacer, de convivir, de percibir que se tiene en el aula y 2) la diversidad misma que debe darse en cuanto a técnicas de enseñanza y estrategias de aprendizaje se refiere sin desconocer la tendencia más marcada en la muestra.

El cruce de resultados de ambos inventarios pone de presente la coincidencia en el estilo reflexivo (lógicos, analíticos) de la muestra mayoritaria de estudiantes (Felder y Silverman: 59\%, EA activo-reflexivo; Herrmann: 76\% PN al estilo CI, cuya característica esencial es ser reflexivo). Esta coincidencia es además reforzada por el porcentaje de $59 \%$ con EA entre lo secuencial y lo global y el $27 \%$ de estudiantes que presenta PN al estilo LI, los cuales son reflexivos, metódicos y secuenciales.

El estilo global se puede relacionar con el $36.1 \%$ de estudiantes que presenta una PN por el estilo CD (holís- ticos, intuitivos, integradores, sintetizadores, prefieren la visualización y las imágenes) y con los que presen$\tan$ EA entre lo visual y lo verbal (39\%) y aquellos que tienen una preferencia fuerte (PF) a lo visual (15\%). De igual manera, el estilo LD se puede relacionar con el porcentaje de estudiantes que son activos (59\%) y gustan de integrar los aprendizajes por la acción y la experiencia. Asimismo el 39\% de estudiantes con EA entre lo visual y verbal permite relacionar a los verbales con el 39\% de LD, competentes en la expresión oral y escrita.

Adicionalmente, después de socializar con los estudiantes los resultados del diagnóstico de estilos de aprendizaje según los modelos de Felder y Silverman y de Herrmann, se les aplicó una encuesta para cotejar tales resultados con la visión que los propios estudiantes tienen de su(s) estilo(s) de aprendizaje. En este sentido, se les preguntó si se sentían identificados con el (los) estilo(s) de aprendizaje arrojado(s) por el inventario de Felder y Silverman y el de Herrmann. A lo cual, 67\% y $30 \%$ respondieron estar, respectivamente, de acuerdo y totalmente de acuerdo con los resultados del inventario de Felder y Silverman. Con respecto al inventario de Herrmann, un $77 \%$ y un $22 \%$ manifestaron estar, res- 
pectivamente, de acuerdo y totalmente de acuerdo con los resultados obtenidos. Estos resultados evidencian el alto grado de satisfacción de los estudiantes con respecto a los resultados del diagnóstico, lo cual refleja también la validez de los instrumentos aplicados, en el sentido planteado, por Hernández y otros (2010), de medir realmente la variable que pretender medir, en este caso, los estilos de aprendizaje.

Inventario de estilo personal de docentes según Herrmann

Por otra parte, los resultados del inventario de Herrmann aplicado a los docentes de los cursos básicos de inglés y

Tabla 3. Resultados del inventario de estilo personal de enseñanza según Herrmann.

\begin{tabular}{|l|c|c|c|c|}
\hline \multicolumn{1}{|c|}{ Estilos de aprendizaje } & $\begin{array}{c}\text { Cortical } \\
\text { izquierdo }\end{array}$ & $\begin{array}{c}\text { Límbico } \\
\text { izquierdo }\end{array}$ & $\begin{array}{c}\text { Límbico } \\
\text { derecho }\end{array}$ & $\begin{array}{c}\text { Cortical } \\
\text { derecho }\end{array}$ \\
\hline $\begin{array}{l}16 \text { docentes de Lenguas } \\
\text { Modernas }\end{array}$ & $56 \%\left(\mathrm{PN}^{*}\right)$ & $37 \%(\mathrm{PI})$ & $68 \%(\mathrm{PN})$ & $50 \%(\mathrm{NP})$ \\
\hline
\end{tabular}

${ }^{*}$ PN: preferencia neta, PI: preferencia intermedia, NP: no preferencia.

francés para conocer su estilo personal como profesional de la enseñanza evidencian un $68 \%$ de docentes con PN al estilo LD, $56 \%$ de docentes con PN al estilo CI, un $37 \%$ con PM al estilo LI y un 50\% de docentes que no tienen preferencia por el estilo CD. Si comparamos estos resultados con los perfiles obtenidos por los estudiantes según el inventario de Herrmann encontramos que mientras la mayoría de profesores $(68 \%)$ tienen un estilo personal de enseñanza con $\mathrm{PN}$ en el cuadrante LD, la mayoría de estudiantes $(76 \%)$ presenta un estilo de aprendizaje con PN en el cuadrante CI (frente a un $56 \%$ de docentes con PN a ese estilo), seguido de un $50 \%$ con preferencia intermedia-PI al estilo LI (frente a $37 \%$ de docentes con PI al estilo LI). Es de resaltar que sólo un 39\% de los estudiantes coincide con los docentes mayoritariamente $(68 \%)$ con PN al estilo LD (Ver Tabla 3).

Estos resultados indican comparativamente que mientras los estudiantes CI tienden mayoritariamente a ser intuitivos, animosos, soñadores, gustan de proyectos originales, van a lo esencial, tienen visión futurista, detestan lo tradicional, son experimentales y críticos, esperan resultados, los docentes LD en su mayoría tienden a un estilo personal de enseñanza que hace énfasis en la comunicación y las relaciones humanas, la mediación, el buen ambiente de la clase, la disciplina, el debate, el trabajo en equipo, la evaluación formativa, el saber hacer más que el contenido. Un docente, así: es tradicional, le disgustan los ejercicios originales o fantásticos; es muy paternalista, desarrolla dependencias afectivas con el grupo, es instintivo, subjetivo, parcial, disperso, poco crítico, hace énfasis en lo oral, tiene en cuenta más el comportamiento que los conocimientos.

Se encontró entonces dos estilos muy diferentes, uno de enseñanza y otro de aprendizaje, lo cual a nivel pedagógico tiene efectos, a veces negativos, si el docente no es consciente de las diferencias entre ambos estilos y no trata de reformular el curso de la clase para atender a las necesidades de aprendizaje del perfil mayoritario en sus estudiantes, sin descuidar eso sí, los otros estilos presentes en el grupo en menores porcentajes.

Los anteriores resultados y las tipificaciones y caracterizaciones de cada estilo dejan de manifiesto la heterogeneidad de la muestra y la disimilitud entre los estilos de enseñanza de los docentes y los estilos de aprendizaje de los estudiantes del Programa de Lenguas de la Universidad del Quindío participantes en esta investigación.

Comparación de resultados de los inventarios de Felder y Silverman y el de Herrmann en otras investigaciones

Para indagar sobre el comportamiento de los resultados de los inventarios de estilos de aprendizaje de Felder y Silverman y de Herrmann en otras investigaciones y compararlos con los arrojados por esta investigación se presentan a continuación los siguientes datos.

Con relación al inventario de Felder y Silverman, los resultados arrojados por esta investigación coinciden con resultados obtenidos en otras investigaciones en programas universitarios de lenguas extranjeras, ingenierías, sistemas de información, ciencias sociales en varios 
países hispanohablantes (Durán y Costaguta, 2007; Mendoza, 2006; Figueroa y otros, 2005, citados en Felder y Spurlin 2005). En ellas se coincide en la dominancia de un equilibrio apropiado en diferentes dimensiones: activo-reflexivo, sensitivo-intuitivo, visual-verbal y secuencial-global, y en la dominancia de los estilos en a de las dimensiones bipolares establecidas por Felder y Silverman: activo, intuitivo, visual y secuencial. Este análisis también es comparable con los datos de algunas investigaciones en Estados Unidos y en Brasil (Constant,1997; Lopes, 2002; Rosati, 1999, citados en Felder y Spurlin, 2005) en cuyas investigaciones se mantiene la tendencia preferencial a los estilos activo, sensitivo, visual y secuencial. Estos resultados podrían indicar, en los estudiantes universitarios examinados, una tendencia a preferir procesos de aprendizaje en los cuales la reflexión y la acción son muy importantes (equilibrio apropiado), claro que con una mejor respuesta a metodologías activas e interactivas.

Asimismo, comparando los resultados de la aplicación del cuestionario de Herrmann en esta investigación con otras a nivel latinoamericano (Velásquez y otros, 2007; Torres y Lajo, 2009) puede decirse que coinciden en las dominancias en los cuadrantes CI y LD.

En resumen, las anteriores comparaciones nos muestran que los resultados obtenidos en la aplicación de los cuestionarios siguen unas tendencias generales que contribuyen a la validación y confiabilidad de tales instrumentos de identificación de estilos de aprendizaje.

\section{Lineamientos para la estructuración de una pro- puesta pedagógica y curricular}

Diferentes fórmulas han sido planteadas desde la teoría de los estilos de aprendizaje para reformular el quehacer pedagógico y mejorar el proceso y los resultados de aprendizaje, resaltando la importancia de aprender a aprender, del aprendizaje para toda la vida. Alonso y otros (1994) identifican tres componentes en la idea de aprender a aprender: las necesidades del estudiante (las competencias a desarrollar), su(s) estilo(s) de aprendizaje y la formación. Estos tres componentes se toman en cuenta para la estructuración de esta propuesta considerándose como necesidades las competencias establecidas desde la Misión, la Visión y los microcurrículos
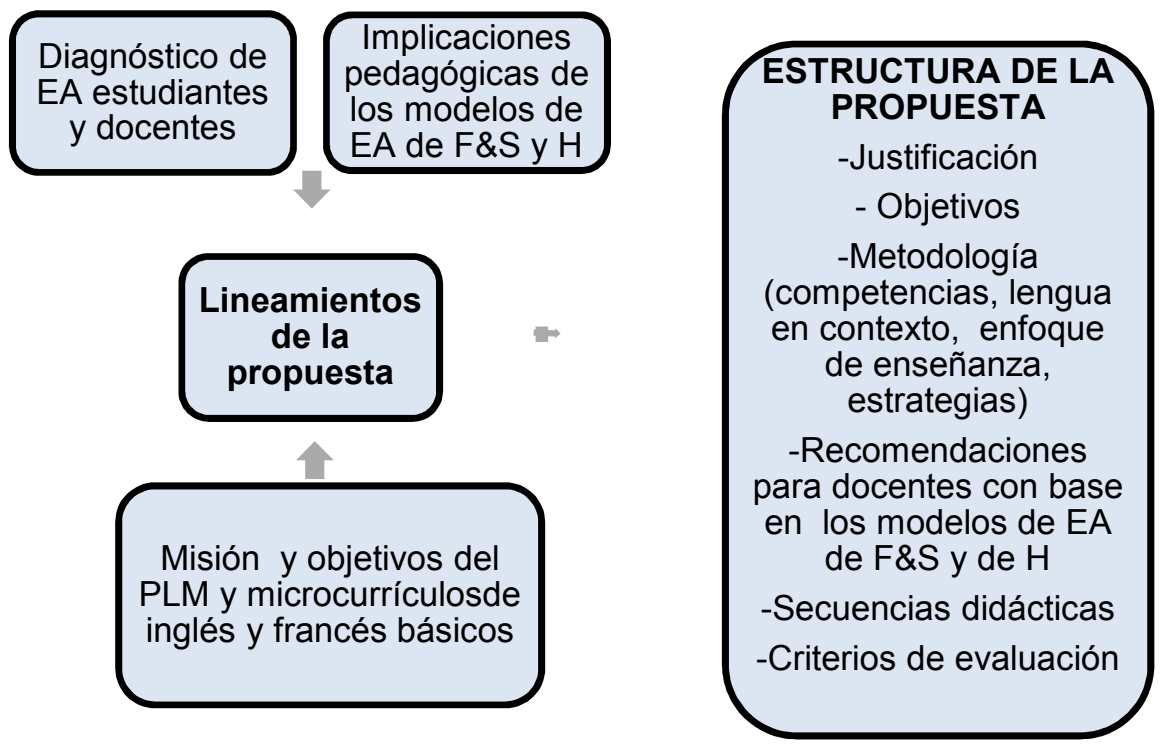

Figura 1. Estructura de la propuesta

de inglés y de francés del Programa de Lenguas Modernas, en relación con los resultados obtenidos de la aplicación de los cuestionarios de estilos de aprendizaje de Herrmann y Felder y Silverman y de los de estilo personal de enseñanza de docentes. De estos dos componentes surge, en el contexto de la formación, la intervención del docente con miras a optimizar los procesos de enseñanza y aprendizaje de las lenguas extranjeras en cuestión.
A continuación se presenta la estructura de la propuesta pedagógica producto de esta investigación (Ver figura 1).

En este sentido, la propuesta se fundamentaría en tales lineamientos y su estructuración contemplaría los siguientes elementos: justificación, objetivos, metodología, secuencias didácticas, recomendaciones para docentes y estudiantes, criterios de evaluación. La consolidación de la propuesta y su implementación hacen 
parte de una investigación de acción educativa a desarrollarse en posteriores procesos investigativos.

Los lineamientos de la propuesta se presentan brevemente a continuación:

\section{En cuanto a la Metodología}

- Un enfoque metodológico donde se equilibre la estructura con la innovación, la formalidad con la informalidad, la teoría con la experiencia, la planificación con la espontaneidad, la reflexión con la acción.

- La implementación de técnicas y estrategias que integren ambientes en los cuales haya espacio para la innovación, la creatividad, la intuición, la proyección, la iniciativa y la espontaneidad bajo parámetros claros en términos de organización, planeación y objetivos. Con esto se logra que los estudiantes se sientan cómodos con las actividades mientras desarrollan cierto grado de tolerancia y ejercitan otros estilos de aprendizaje en los cuales son menos experimentados.

- Un equilibrio entre teoría, leyes, reglas, hechos, diagramas, etc. y la interacción, la cooperación, los ambientes cálidos, espontáneos, acogedores, en los cuales se brinde una retroalimentación constante y respetuosa y se valore la experiencia y los gustos personales y se garantice variedad de estrategias y actividades pedagógicas. Es decir equilibrar lo lógico y lo emocional.

- La implementación de técnicas de aprendizaje cooperativo y de pedagogía por proyectos.

- La utilización de esquemas deductivos en los cuales se promueva la intuición y la reflexión.

- El desarrollo de habilidades sociales que promuevan valores como el respeto y el aprecio por las opiniones, las experiencias y los gustos personales.

- La constante y respetuosa retroalimentación de los procesos de aprendizaje.

- La utilización de variedad de estrategias pedagógicas tales como proyectos, juegos, visitas y salidas pedagógicas, música, estímulos visuales y dramatizaciones.

En cuanto al actuar y comportamiento de los docentes se recomienda:

- Equilibrar el grado de estructuración e innovación, la formalidad y la informalidad en la clase.

- Preocuparse por la forma como se imparten y se reciben los conocimientos, más allá de los contenidos.

- Recurrir más a actividades comunicativas, lúdicas y cooperativas que permitan distensionar el ambiente y promuevan la adquisición de saberes y desarrollo de habilidades sin descuidar la rigurosidad de los procedimientos y la profundidad de los conocimientos.

- Ser lúdicos, entusiastas, mediadores, facilitadores, comprensivos, abiertos al diálogo, partidarios del trabajo en equipo, la interdisciplinariedad y la expresión oral.

- Entablar relaciones de respeto y consideración sin crear dependencias afectivas con los estudiantes.

- Ser más objetivos en la evaluación de los estudiantes, y equilibrar la importancia que dan al progreso del estudiante y a la calidad del producto, al comportamiento y al desempeño.

- Evaluar tanto las actitudes como las aptitudes de los estudiantes haciendo énfasis en las actividades comunicativas y cooperativas, dando igual importancia tanto a la producción oral como a las demás habilidades comunicativas: escritura, lectura, escucha.

\section{CONCLUSIONES}

- La investigación produjo un diagnóstico que confirmó una ligera tendencia a la reflexión y la heterogeneidad en los estilos de aprendizaje de los estudiantes de los cursos básicos de inglés y francés del Programa de Lenguas Modernas de la Universidad del Quindío. Además, el diagnóstico de los estilos de enseñanza confirmó la disimilitud entre los estilos de aprendizaje de los estudiantes y los estilos de enseñanza de los docentes, ya que, según el inventario de Herrmann, la mayoría de profesores tienen un estilo personal de enseñanza predominante en LD mientras la mayoría de estudiantes se inclinan fuertemente al CI.

- Comparados los resultados de esta investigación con los obtenidos en otras investigaciones iberoamericanas, se encuentran coincidencias en la tendencia generalizada de los estudiantes a un equilibrio apropiado entre activo y reflexivo, sensitivo-intuitivo y secuencial- global. También hay coincidencia en los mayores porcentajes ubicados en los cuadrantes CI y LD. En cuanto al estilo personal de enseñanza, también se coincide en la disimilitud entre estos y los estilos de aprendizaje de los estudiantes.

- El diagnóstico de estilos de aprendizaje y de enseñanza implica en el contexto pedagógico y curricular la variedad, la innovación y el equilibrio del docente para que, a medida que éste explora en otros 
estilos diferentes a sus tendencias más marcadas, fortalezca los estilos de aprendizaje de sus estudiantes, les motive a explorar y desarrollar otros, mientras se optimiza el aprendizaje de las lenguas extranjeras.

- La propuesta pedagógica producto de esta investigación está fundamentada en el diagnóstico y en las implicaciones de los estilos de aprendizaje de estudiantes y de estilos de enseñanza de docentes, de acuerdo, además, con los objetivos planteados desde la misión, la visión y los microcurrículos de los cursos básicos de inglés y de francés en el Programa de Lenguas Modernas de la Universidad del Quindío. Esta propuesta será implementada en posteriores investigaciones mediante el uso de secuencias didácticas y se espera que impacte positivamente la práctica pedagógica del docente y el aprendizaje de los estudiantes, que contribuya en los procesos de diseño curricular y que pueda ser replicada en contextos similares.

\section{BIBLIOGRAFIA}

1. Alonso, C.M; Gallego, D.J. y Honey, P. (1994) Estilos de Aprendizaje. Qué son. Cómo se diagnostican. Bilbao: Mensajero.

2. Cazau, Pablo (2004). Guía de Estilos de Aprendizaje. Disponible en : http://galeon.hispavista.com/ pcazau/guia_esti.htm $>$ Consultado 15/09/2008.

3. Centeno. A. y otros (2005). "Identificación de estilos de enseñanza en la universidad. Estudio en tres carreras universitarias: ciencias biomédicas, abogacía y comunicación social”. Presentado en el Quinto Coloquio de Gestión Universitaria en América del Sur. Argentina: Universidad Priada de la ciudad autónoma de Buenos Aires, www.austral.edu.ar/oei/archivos/07.pdf. Consultado abril 12 de 2007

4. Chalvin M.J. (1995). Los dos cerebros en el aula, TEA Ediciones, Madrid.

5. Chomsky N. (1980). El lenguaje y el entendimiento. Planeta-Agostini, Barcelona.

6. Dell'Ordine.http://www.monografias.com/trabajos44/estilos-aprendizaje/estilos-aprendizaje2.shtml .2001 (s/p) (consultado en 2011).

7. Dunn, R.; Dunn,K. (1984). La enseñanza y el estilo de aprendizaje. Madrid: Anaya.

8. Felder R. M y Spurlin, J. (2005). Application, reliablility and validity of the index of learning styles. Int. J. Engng. Vol 21, No.1. Printed in Great Britain.

9. Fodor J. (1986) La modularidad de la mente, Ediciones Morata, Madrid.

10. Gómez L. "Estrategia y alternativa pedagógicas", Red maestros de maestros: Programa de apoyo a la docencia, Santiago de Chile. Disponible en: http://www.rmm.cl/index_sub.php?id_ seccion $=1724 \& i d \_p o r t a l=276 \& i d \_c o n t e n i d o=4170$ (consultado en 2011).

11. Hernández R. L. (2004) La importancia de los estilos de aprendizaje en la enseñanza de inglés como lengua extranjera. En Revista de Estudios Literarios. Facultad de Ciencias de la Información. No. 27. Disponible en: https://www.ucm.es/info/especulo/numero27/estilosa.html (consultado en 2011).

12. Guild P. y Garger S. (1998). Marching to Different Drummers. Virginia, USA: ASCD-Association for Supervision and Curriculum Development. 2nd Ed. Publishers London.

13. Hirsh S. y Kummerow J. (1990). Introduction to type in organizations: Individual Interpretive Guide. 2nd Ed., Palo Alto, CA: Consulting Psychologists Press, Inc.

14. Kagan N. y Krathwohl D. (1967). En Alonso, C. (1992a:51). Análisis y Diagnóstico de los Estilos de Aprendizaje en Estudiantes Universitarios. Tomo I. Madrid: Colección Tesis Doctorales. Editorial de la Universidad Complutense.

15. Kindelán M. (1992) Estilos y Estrategias de Aprendizaje de una Segunda Lengua en un Contexto de Inglés Aplicado a la Ciencia y la Tecnología. Archivo PDF. Disponible en: dialnet.unirioja.es/servlet/ fichero_articulo?codigo $=1958134 \&$ orden $=63866$ (consultado en 2011)

16. Krippendorff K. (1990) Metodología del análisis de contenido. Teoría y práctica. Barcelona. Paidós.

17. Labatut E. (2004). Aprendizaje universitario: un enfoque Metacognitivo. Tesis doctoral, ISBN: 84669-2345-4, Madrid. Disponible en http://www.ucm.es/BUCM/tesis/edu/ucm-t27286.pdf (consultado en 2011).

18. Latorre A., del Rincón D. y Arnal J. (1997). Bases metodológicas de la investigación educativa. Barcelona: Hurtado Ediciones. 
19. López, J. M. (1996). Los estilos de aprendizaje y los estilos de enseñanza. Un modelo de categorización de estilos de aprendizaje de los alumnos de enseñanza secundaria desde el punto de vista del profesor. En Revista Anales de Psicología, Monográfico: Estrategias y Estilos de Aprendizaje. 12(2), 179-184.

20. Lozano, A. (2009). Estilos de aprendizaje y enseñanza, un panorama de la estilística educativa. México: Trillas.

21. Maclean P. (1978). Education and the brain. Chicago: Chicago Press.

22. Martínez J. (2002), Tendencias en los estilos de aprendizaje de una lengua extranjera. En Revista Didáctica (Lengua y Literatura) ISSN: 1130-0531, Vol. 14(2002): 175-193

23. Pask G. (1976). Styles and Strategies of Learning. En British Journal of Educational Psychology, 46: $128-148$

24. Pinelo F. T. (2008). Estilos de enseñanza de los profesores de la carrera de psicología. En Revista Mexicana de Orientación Educativa, Vol.5, No.13, pp.17-24.

25. Riding R y Rayner S. (1998). Cognitive Styles and Learning Strategies. Understanding Style Differences in Learning and Behaviour. David Fulton.

26. Rendón M. A. y otros (2010). Informe final los estilos de enseñanza en la Universidad de Antioquia (Primera Fase Facultad de Educación). Medellín: En Revisión.

27. Reyes F. (2008). Estilos de aprendizaje. p.1. Disponible en: http://periplosenred.blogspot. com/2008/11/estilos-de-aprendizaje.html (consultado en 2011).

28. Ruiz Bolívar, C. (2004) Neurociencia y educación. En Neurociencia cognitiva y educación, 140-157. 51-74-97-9775975, Universidad Nacional Pedro Ruiz Gallo. Perú. Disponible en: http://www.psicojack.com/archivos/200109c.pdf. Consultado en 2011.

29. Sperry R. (1973). Lateral specialization of cerebral function in the surgically separated hemispheres. En: F. J. McGuigan (Ed.) .The Psychophisioly of the thinking. New York: Press.

30. Sternberg, R. J. (1977). Intelligence, Information Processing, and Analogical Reasoning. Hillsdale, N. J: Erlbaum.

31. Torres M. y Lajos R. (2009). Dominancia cerebral asociada al desempeño laboral de los docentes de una UGEL de Lima. En Revista IIPSI, Vol. 12. No. 1. Disponible en : http://sisbib.unmsm.edu.pe/BVRevistas/Investigacion_Psicologia/v12_n1/pdf/a07v12n1.pdf. Consultado en 2011.

32. Velásquez B. y otros. (2007). Determinación del perfil de dominancia cerebral o formas de pensamiento de los estudiantes de primer semestre del programa de bacteriología y laboratorio clínico de la Universidad Colegio Mayor de Cundinamarca. Colegio Mayor de Cundinamarca.

Watson (1925). El conductismo, en Burbano Lida, Teorías del aprendizaje. Disponible en http:// www.monografias.com/trabajos13/teapre/teapre.shtml\#al (consultado en 2011) 